\title{
Spasticity in practice (SPACE): an international non-interventional study of botulinum neurotoxin type-A in treatment-naïe subjects with spasticity
}

\author{
Julian Harriss ${ }^{1}$, Nicolas Roche ${ }^{2}$, Carlos Cantú-Brito ${ }^{3}$, Svetlana Khatkova ${ }^{4}$, Patrik Säterö ${ }^{5}$, \\ Susanne Heitmann ${ }^{6}$, Olivier Simon ${ }^{7}$, Christine Kliebe-Frisch ${ }^{8}$, Georg Comes ${ }^{8}$, Wolfgang H. Jost ${ }^{9} 10$ \\ ${ }^{1}$ IRMA: Independent Rehabilitation Medicine Assessment, London, United Kingdom \\ ${ }^{2}$ Hôpital Raymond Poincaré, Garches, France \\ ${ }^{3}$ Instituto Nacional de Ciencias Médicas y Nutrición Salvador Zubirán, Mexico City, Mexico \\ ${ }^{4}$ Federal State Hospital for Treatment and Rehabilitation, Ministry of Health, Moscow, Russia \\ ${ }^{5}$ Sahlgrenska Universitetssjukhuset/Högsbo, Göteborg, Sweden \\ ${ }^{6}$ DKD HELIOS clinic Wiesbaden, Wiesbaden, Germany \\ ${ }^{7}$ formerly of Merz Pharmaceuticals GmbH, Frankfurt am Main, Germany \\ ${ }^{8}$ Merz Pharmaceuticals GmbH, Frankfurt am Main, Germany \\ ${ }^{9}$ Parkinson-Klinik Ortenau, Wolfach, Germany \\ ${ }^{10}$ University of Freiburg, Freiburg/Breisgau, Germany
}

\begin{abstract}
Aim of the study. SPACE, a prospective, non-interventional, open-label, multinational study, investigated physicians' and subjects' assessment of safety, efficacy, and health-related quality of life (HRQoL) following botulinum neurotoxin type-A (BoNT-A) treatment to understand real-world clinical usage for the management of focal and multifocal spasticity.

Clinical rationale for the study. Treatment guidelines recommend the use of BoNT-A for the management of spasticity in adults. This study assessed how physicians use BoNT-A therapy in real-world clinical practice, and provided evidence on long-term safety and efficacy over a period of up to 2 years.

Materials and methods. BoNT treatment-naïve adults with spasticity of any aetiology received any BoNT-A formulation at their physician's discretion, and were observed for $\leq 8$ treatment cycles ( $\leq 2$ years). Daily practice information, physician's global assessments of tolerability and efficacy, and HRQoL were documented. Incidences of adverse drug reactions or all adverse events were documented for non-Mexican subjects and for Mexican subjects, respectively, due to protocol differences based on local regulatory requirements.

Results. A total of 701 subjects were enrolled (safety population; nine countries). Physicians rated the tolerability of BoNT-A as 'very good' or 'good' for $\mathbf{8 8 . 2 - 9 7 . 4 \% ~ o f ~ s u b j e c t s ~ t h r o u g h o u t ~ t h e ~ s t u d y ~ ( s u b j e c t ~ n u m b e r s ~ d e c l i n e d ~ t h r o u g h o u t ~ t h i s ~ n o n - i n t e r v e n - ~}$ tional study). Adverse drug reactions were reported for $16 / 600$ (2.7\%) of the non-Mexican subjects, with two considered to be 'definitely related' to treatment (injection-site haematoma, $n=1$; botulism, $n=1$ ). For 687 subjects, efficacy was rated'very good' or'good' by most physicians and subjects. Improvements in HRQoL were observed.

Conclusions and clinical implications. Throughout this 2-year study, BoNT-A treatment was generally well-tolerated, effective, and associated with an improved HRQoL. This study makes a valuable contribution to the broader understanding of how physicians use BoNT-A therapy to manage spasticity in real-world clinical practice.
\end{abstract}

Key words: botulinum neurotoxin $A$, rehabilitation, muscle spasticity, quality of life

(Neurol Neurochir Pol 2021; 55 (2): 165-173)

Address for correspondence: Dr Julian Harriss. IRMA, 10 Oakway, Bromley, BR2 0LJ, United Kingdom, e-mail: jharriss@doctors.org.uk 


\section{Introduction}

European and US treatment guidelines and consensus statements recommend intramuscular botulinum neurotoxin type-A (BoNT-A) injections for the management of spasticity in adults [1-4]. At the time when this study was being conducted, three BoNT-A formulations were commercially available in North America and Europe: incobotulinumtoxin A (Xeomin ${ }^{\circledR}$; Merz Pharmaceuticals GmbH, Frankfurt am Main, Germany) $[5,6]$, onabotulinumtoxin A (Botox ${ }^{\mathbb{R}}$; Allergan Inc., Irvine, CA, USA) $[7,8]$, and abobotulinumtoxinA (Dysport ${ }^{\circledR}$; Ipsen Ltd, Boulogne-Billancourt, France) $[9,10]$. Multiple controlled clinical trials have demonstrated the safety and efficacy of repeated BoNT-A injections for focal and multifocal spasticity [11-20]. However, less data exist regarding the long-term safety and efficacy outcomes in real-world clinical practice in subjects with spasticity [21-24].

The SPAsticity in PractiCE (SPACE) study is one of the most extensive non-interventional studies of BoNT-A in spasticity to date, enrolling subjects with spasticity of any aetiology and allowing the treatment of upper- and lower-limb spasticity simultaneously, according to the local approval status of the BoNT-A formulation and clinical setting in participating countries. The study was designed to investigate physicians' and subjects' assessment of safety, efficacy, and health-related quality of life (HRQoL) following BoNT-A treatment of treatment-naïve subjects in routine clinical practice, by collecting data on subjects' disease course and treatments, the treating physicians, and their treatment preferences.

\section{Clinical rationale for the study}

With a paucity of data on the long-term safety and efficacy outcomes related to everyday use of BoNT-A in subjects with spasticity, the primary objective of this study was to investigate physicians' and subjects' assessment of safety, efficacy, and HRQoL in treatment-naïve subjects with multifocal spasticity who received BoNT-A in routine, real-world clinical practice over a treatment period of $\leq 2$ years.

\section{Materials and methods}

\section{Study design and participants}

SPACE was a prospective, non-interventional, open-label, multicentre study conducted in nine countries: Canada, France, Germany, Italy, Mexico, Russia, Spain, Sweden, and the UK. Subjects $\geq 18$ years of age were eligible if they had spasticity of any aetiology requiring BoNT-A injections and had never previously received BoNT-A or -B for any indication. Subjects were also required to have sufficient understanding of the primary local language to complete the study questionnaires and provide informed consent. Subjects already participating in an interventional study, or who were planning to participate in a study involving BoNT-A treatment, were ineligible.
Subjects could receive treatment for $\leq 2$ years with any BoNT-A product available in their country, i.e. incobotulinumtoxinA, onabotulinumtoxinA or abobotulinumtoxinA, according to the local product approval status and the individual subject's needs, at the treating physician's discretion. Therefore, the visit schedule comprised $\leq 8$ injection visits (treatment cycles) at intervals $>3$ months, plus a final visit with no injection in subjects who returned for assessment at the end of the study. Doses (total and per-muscle), injection sites, injection techniques, and treatment intervals were determined by each physician based on clinical need.

The study was conducted in accordance with the ethical principles of the Declaration of Helsinki and in compliance with local regulatory requirements. Study documents were reviewed and approved by Ethics Committees and regulatory authorities according to local requirements in the participating countries. Subjects had to provide written informed consent, in the local language, for the use of their data or, if unable to sign the consent form, could consent verbally in the presence of a witness. The study protocol was registered with the Verband Forschender Pharma-Unternehmen Deutschland (the Association of Pharmaceutical Research Companies of Germany), https://www.vfa.de/de/ arzneimittel-forschung/datenbanken-zu-arzneimitteln/nisdb/ nis-details/_533.

\section{Daily practice information}

Information was captured on participating subjects, including history and current spasticity status (e.g. aetiology, topography, patterns of spasticity), details of each BoNT-A treatment (BoNT-A formulation used, muscles treated, injection sites/muscle, assessment scales used, injection guidance [e.g. electromyography with or without electrostimulation, ultrasound]), and concomitant treatment.

Data were also collected on treating physicians, including their medical speciality, years in clinical practice, previous experience with BoNT-A, and opinions on dosages.

\section{Safety}

Physicians rated each subject's tolerability of the medication at the end of each treatment cycle (i.e. at the next injection visit) on a 4-point scale from 1 (very good) to 4 (poor).

The incidence of adverse drug reactions (ADRs, defined as adverse events [AEs] for which a causal relationship to treatment cannot be excluded) was reported for all subjects enrolled, including subjects with no record of the BoNT-A product administered at the first injection, but for whom product data had been recorded at subsequent injections. In Mexico, due to local regulatory requirements, the incidence of all AEs (serious and non-serious, related or not), including ADRs, was recorded using a specific ADR/AE reporting form. As non-related AEs were recorded in addition to ADRs in Mexico, these were analysed separately from ADRs in non-Mexican countries. 


\section{Global assessment of efficacy}

Physicians could document treatment efficacy using various impairment- or function-based scales (e.g. Ashworth Scale, Tardieu Scale, Rivermead Scale, Functional Ambulation Classification Scale). However, in this diverse, multinational study population, efficacy assessments were not consistently performed by all physicians in routine clinical practice. Therefore, global assessments of efficacy relevant to real-world clinical practice were assessed, and are reported here.

Physicians and subjects rated the efficacy of each treatment at the end of each treatment cycle (i.e. at the next injection visit) on a 4-point scale from 1 (very good) to 4 (poor). Responders were subjects with a score $\leq 3$ (at least moderate efficacy).

\section{HRQoL}

HRQoL was assessed using the EuroQoL 5-dimension questionnaire (EQ-5D) visual analogue scale (VAS) [25], completed by the subjects at their study centres during each injection visit and at home 4 weeks post injection (i.e. during the assumed peak effect of treatment [26]). Subjects rated their current state of health on a quantitative scale from 0 (the worst imaginable) to 100 (the best imaginable).

Subjects also selected the statement that best described their state of health on that day using the five dimensions of the EQ-5D descriptive system (mobility, self-care, usual activities, pain/discomfort, and anxiety/depression), and completed the 12-item Short-Form (SF-12) Health Survey to describe their perceived state of health (see Supplemental Methods).

\section{Statistical analysis}

Study data were summarised using descriptive statistics. All subjects enrolled were analysed for safety. All subjects with an available date of written informed consent and recorded information about the BoNT-A product administered at the first injection were analysed for efficacy and HRQoL. Subjects with no reported BoNT-A product at their first injection, who could not therefore be assigned to a treatment group (incobotulinumtoxinA, onabotulinumtoxinA, or abobotulinumtoxinA), were excluded from the efficacy and HRQoL analyses. Missing values were not imputed, and all analyses were conducted on observed cases.

Two post-hoc analyses were performed. One assessed differences in the proportion of non-Mexican subjects with $\geq 1$ ADR or serious ADR (SADR) between treatment groups using Fisher's exact test. Another post-hoc analysis assessed change from Visit 1 (baseline) in EQ-5D VAS score using the Wilcoxon signed rank test; in this analysis, data following a switch between treatment groups was excluded.

\section{Results}

\section{Daily practice information Subjects}

A total of 701 subjects were evaluated for safety; efficacy and HRQoL outcomes were assessed in 687 subjects with an available date of written consent and reported BoNT-A product administered at the first injection (Supplemental Fig. 1). Subjects' mean (standard deviation [SD]) age was 55.0 (15.5) years, and $61.3 \%$ were male (Tab. 1). The median (range) time since onset of spastic symptoms was 2.0 (0-79) years. Most participants had post-stroke spasticity and paresis most commonly presented as hemiplegia. At least one relevant concomitant medication was documented for $36.8 \%$ of subjects, among which antithrombotic agents (15.6\%), muscle relaxants (13.4\%), and lipid-modifying agents (13.0\%) were the most frequently documented. At least one concomitant therapy was documented in $23.7 \%$ of subjects, with physiotherapy $(20.7 \%)$ and occupational therapy $(12.7 \%)$ the most frequently documented.

Overall, 205/701 (29.2\%) subjects formally discontinued from the study prematurely, including four with no reported BoNT-A product at their first injection (Supplemental Fig. 1). The main reasons for discontinuation were: loss to follow-up (74/701, 10.6\%), lack of efficacy $(28 / 701,4.0 \%)$, and another undocumented reason $(79 / 701,11.3 \%)$. The proportion of subjects attending at each injection visit also decreased throughout the study (Supplemental Tab. 1), although reasons were not documented.

The median (interquartile range; IQR) injection interval ranged from $3.5(3.0-4.6)$ months at Visit 2 to $3.2(3.0-3.7)$ months at Visit 8, with 22.8 (21.4-26.3) months between the first and last injections. The most frequently injected upper-limb muscles at Visit 1 were the flexor digitorum superficialis, biceps brachii, and flexor digitorum profundus (Supplemental Fig. 2a). The most frequently injected lower-limb muscles were the gastrocnemius caput mediale, soleus, gastrocnemius caput laterale, and tibialis posterior (Supplemental Fig. 2c). These remained among the most frequently injected muscles at Visit 8 (Supplemental Fig. 2b and d).

The median (IQR) total incobotulinumtoxinA, onabotulinumtoxinA, and abobotulinumtoxinA doses administered at Visit 1 were $30(20,50) \mathrm{U}, 50(40,75) \mathrm{U}$, and $125(100,200) \mathrm{U}$. Median doses administered into the most frequently injected muscles at Visit 1 are summarised in Table 1. The treating physician performed the BoNT-A injection for most subjects (67.9\% of those with data recorded; 411/605) at Visit 1.

For $40.5 \%$ of subjects with data recorded, injections were administered without the use of guidance techniques at Visit 1 $(47.6 \%, 19.0 \%$, and $37.5 \%$ with incobotulinumtoxinA, onabotulinumtoxin A, and abobotulinumtoxin A, respectively). Electrostimulation was used in $18.6 \%(17.4 \%, 23.9 \%$, and $16.3 \%$, respectively), electromyography in $14.8 \%(12.4 \%, 17.6 \%$, and 
Table 1. Subject baseline demographics and characteristics

\begin{tabular}{|c|c|c|c|c|}
\hline Characteristic & $\begin{array}{l}\text { Incobotulinum- } \\
\text { toxinA } \\
n=465\end{array}$ & $\begin{array}{l}\text { Onabotulinum- } \\
\text { toxinA } \\
n=142\end{array}$ & $\begin{array}{l}\text { Abobotulinum- } \\
\text { toxinA } \\
\mathbf{n}=\mathbf{8 0}\end{array}$ & $\begin{array}{c}\text { Study } \\
\text { population } \\
n=687\end{array}$ \\
\hline Male sex, n (\%) & $283(60.9)$ & $83(58.5)$ & $55(68.8)$ & $421(61.3)$ \\
\hline Age, years; mean (SD) & $54.6(15.6)$ & $55.6(15.3)$ & $56.2(15.3)$ & $55.0(15.5)$ \\
\hline $\mathrm{BMI}, \mathrm{kg} / \mathrm{m}^{2} ;$ mean $(\mathrm{SD})$ & $25.8(5.1)$ & $25.6(5.7)$ & $25.7(4.6)$ & $25.7(5.1)$ \\
\hline Time since spasticity-causing event, years; median (range) & $2.0(0-63)$ & $2.0(0-52)$ & $2.0(0-55)$ & $2.0(0-63)$ \\
\hline Time since onset of spastic symptoms, years; median (range) & $2.0(0-59)$ & $2.0(0-51)$ & $2.0(0-79)$ & $2.0(0-79)$ \\
\hline \multicolumn{5}{|l|}{ Aetiology of spasticity, $\mathrm{n}(\%)^{\mathrm{a}}$} \\
\hline Stroke & $310(66.7)$ & $85(59.9)$ & $49(61.3)$ & $444(64.6)$ \\
\hline Brain injury & $33(7.1)$ & $4(2.8)$ & $4(5.0)$ & $41(6.0)$ \\
\hline Multiple sclerosis & $36(7.7)$ & $19(13.4)$ & $9(11.3)$ & $64(9.3)$ \\
\hline Spinal-cord injury & $19(4.1)$ & $8(5.6)$ & $3(3.8)$ & $30(4.4)$ \\
\hline Cerebral palsy & $10(2.2)$ & $4(2.8)$ & $4(5.0)$ & $18(2.6)$ \\
\hline Other & $48(10.3)$ & $18(12.7)$ & $11(13.8)$ & $77(11.2)$ \\
\hline Missing & $2(0.4)$ & 0 & 0 & $2(0.3)$ \\
\hline \multicolumn{5}{|l|}{ Topographical distribution of paresis, $\mathbf{n}(\%)$} \\
\hline Hemiplegia & $377(81.1)$ & $106(74.6)$ & $58(72.5)$ & $541(78.7)$ \\
\hline Diplegia & $44(9.5)$ & $23(16.2)$ & $12(15.0)$ & $79(11.5)$ \\
\hline Quadriplegia & $41(8.8)$ & $9(6.3)$ & $8(10.0)$ & $58(8.4)$ \\
\hline Missing & $3(0.6)$ & $4(2.8)$ & $2(2.5)$ & $9(1.3)$ \\
\hline \multicolumn{5}{|c|}{ BoNT-A dose in most frequently injected muscles at Visit 1, median (IQR) } \\
\hline Overall, median (IQR) [n] & $30(20,50)[2,899]$ & $50(40,75)[676]$ & $125(100,200)[476]$ & NA \\
\hline Upper limb & & & & NA \\
\hline Flexor digitorum superficialis; $L, R$ & $\begin{array}{l}30(25,50) \\
30(25,50)\end{array}$ & $\begin{array}{c}55(40,77.5) \\
60(50,75)\end{array}$ & $\begin{array}{l}175(100,250) \\
150(100,200)\end{array}$ & \\
\hline Biceps brachii: L, R & $\begin{array}{l}60(40,75) \\
50(40,75)\end{array}$ & $\begin{array}{c}50(50,75) \\
55(50,77.5)\end{array}$ & $\begin{array}{l}225(150,300) \\
150(100,200)\end{array}$ & \\
\hline Flexor digitorum profundus: L, R & $\begin{array}{l}30(20,40) \\
25(20,40)\end{array}$ & $\begin{array}{l}50(50,70) \\
50(50,60)\end{array}$ & $\begin{array}{c}150(100,200) \\
100(50,200)\end{array}$ & \\
\hline Lower limb & & & & NA \\
\hline Gastrocnemius caput mediale; L, R & $\begin{array}{l}50(40,55) \\
50(35,50)\end{array}$ & $\begin{array}{l}50(40,75), \\
50(40,75)\end{array}$ & $\begin{array}{l}150(100,250) \\
125(100,150)\end{array}$ & \\
\hline Soleus; L, R & $\begin{array}{l}50(40,70) \\
50(50,75)\end{array}$ & $\begin{array}{l}60(50,85) \\
70(50,80)\end{array}$ & $\begin{array}{l}200(150,200) \\
150(125,200)\end{array}$ & \\
\hline Gastrocnemius caput laterale; L, R & $\begin{array}{l}50(40,50) \\
50(35,50)\end{array}$ & $\begin{array}{l}50(40,75) \\
50(40,75)\end{array}$ & $\begin{array}{c}100(80,125) \\
125(50,150)\end{array}$ & \\
\hline
\end{tabular}

$23.8 \%$, respectively), and ultrasound in $13.6 \%$ (14.6\%, $11.3 \%$, and $12.5 \%$, respectively) of subjects.

\section{Physicians}

Of the 171 participating physicians, $54(31.6 \%)$ were from Germany, 33 (19.3\%) from France, 28 (16.4\%) from Italy, 14 (8.2\%) from the UK, 11 (6.4\%) from Russia, $11(6.4 \%)$ from Spain, 10 (5.8\%) from Canada, seven (4.1\%) from Mexico, and three (1.8\%) from Sweden. Most were neurologists $(47.4 \%)$ or physiatrists $(28.1 \%)$. The physicians had a mean (SD) of 15.7 (8.7) years of experience in medical practice and a mean (SD) of 9.2 (5.8) years of experience with BoNT injections; 59.1\% stated that they would like to inject higher doses of BoNT-A than permitted by current product labelling. The mean (SD; IQR) higher doses these physicians would like to inject were incobotulinumtoxinA $651.8 \mathrm{U}(191.6 ; 600-800)$, onabotulinumtoxinA $640.3 \mathrm{U}(170.4 ; 500-800)$, and abobotulinumtoxin A 1,751.9 U (844.2; 1,500-2,000).

\section{Safety}

Safety analyses were performed for all 701 subjects across the entire study duration ( $\leq 2$ years). The tolerability of all BoNT-A formulations was rated as 'very good' or 'good' by 
Table 2. Frequency of non-Mexican subjects with ADRs

\begin{tabular}{|c|c|c|c|c|}
\hline System organ class & $\begin{array}{l}\text { Incobotulinum- } \\
\text { toxinA } \\
n=369\end{array}$ & $\begin{array}{l}\text { Onabotulinum- } \\
\text { toxinA } \\
n=142\end{array}$ & $\begin{array}{l}\text { Abobotulinum- } \\
\text { toxinA } \\
n=75\end{array}$ & $\begin{array}{c}\text { Total } \\
n=600\end{array}$ \\
\hline Subjects with $\geq 1$ ADR, $n$ (\%) & $11(3.0)$ & $3(2.1)$ & $2(2.7)$ & $16(2.7)$ \\
\hline General disorders and administration-site conditions & $3(0.8)$ & $1(0.7)$ & $1(1.3)$ & $5(0.8)$ \\
\hline Nervous system disorders & $4(1.1)$ & $1(0.7)$ & $0(0.0)$ & $5(0.8)$ \\
\hline Gastrointestinal disorders & $1(0.3)$ & $0(0.0)$ & $1(1.3)$ & $2(0.3)$ \\
\hline Infections and infestations & $1(0.3)$ & $0(0.0)$ & $1(1.3)$ & $2(0.3)$ \\
\hline Eye disorders & $0(0.0)$ & $0(0.0)$ & $1(1.3)$ & $1(0.2)$ \\
\hline Injury, poisoning, and procedural complications & $1(0.3)$ & $0(0.0)$ & $0(0.0)$ & $1(0.2)$ \\
\hline Investigations & $1(0.3)$ & $0(0.0)$ & $0(0.0)$ & $1(0.2)$ \\
\hline Musculoskeletal and connective tissue disorders & $0(0.0)$ & $1(0.7)$ & $0(0.0)$ & $1(0.2)$ \\
\hline $\begin{array}{l}\text { Neoplasms benign, malignant, and unspecified } \\
\text { (including cysts and polyps) }\end{array}$ & $1(0.3)$ & $0(0.0)$ & $0(0.0)$ & $1(0.2)$ \\
\hline Psychiatric disorders & $0(0.0)$ & $0(0.0)$ & $1(1.3)$ & $1(0.2)$ \\
\hline Respiratory, thoracic, and mediastinal disorders & $1(0.3)$ & $0(0.0)$ & $0(0.0)$ & $1(0.2)$ \\
\hline
\end{tabular}

Data shown for non-Mexican subject population, where incidence of ADRs was documented. In Mexico, the incidences of ADRs and all other AEs, regardless of their relationship with the study treatment, were recorded. Total population includes those with no reported injection in first treatment cycle. Medical terms are as per the Medical Dictionary for Regulatory Activities version 16.0

$\mathrm{ADR}$ - adverse drug reaction; $\mathrm{AE}-$ adverse event; $\mathrm{n}$ - number of subjects; $\mathrm{N}-$ total number of subjects

physicians for the large majority of subjects (88.2-97.4\%) throughout the study (Supplemental Fig. 3).

In most countries, the incidence of ADRs was recorded except in Mexico, where non-related AEs were recorded in addition to ADRs. Therefore, safety outcomes for Mexican $(\mathrm{n}=101)$ and non-Mexican $(\mathrm{n}=600)$ subjects were analysed separately. Among the 600 non-Mexican subjects, over the entire duration of the study, there were only 27 ADRs reported by 16 subjects (Tab. 2). The most frequent system organ classes affected were 'general disorders and administration site conditions' and 'nervous system disorders'. The proportion of subjects with ADRs was similar across the BoNT-A treatment groups (Tab. 2), and post-hoc analysis did not reveal a significant difference ( $\mathrm{p} \geq 0.7666$ for comparisons between treatment groups).

For 8/600 (1.3\%) non-Mexican subjects, at least one ADR was considered 'unlikely to be related', for 2/600 (0.3\%) 'possibly related', for $4 / 600(0.7 \%)$ 'probably related', and for $2 / 600(0.3 \%)$ 'definitely related' to the study medication. The ADRs considered 'definitely related' to the study medication were injection-site haematoma $(n=1)$ and botulism $(n=1)$ that was further described as asthenia, generalised weakness, and a decrease in the activities of daily living.

SADRs were reported for eight subjects; in four subjects these were classed as 'nervous system disorders'. All reported SADRs were considered 'unlikely to be related' to BoNT-A treatment. SADRs included abdominal pain, anxiety, astrocytoma, bipolar disorder, cerebrovascular accident, chronic obstructive pulmonary disease (COPD), death, epilepsy, fall, hemiparesis, hemiplegia, patella fracture, radius fracture, and subarachnoid haemorrhage. The percentage of subjects with SADRs was similar across the three treatment groups
(incobotulinumtoxinA, 5/369 subjects [1.4\%]; onabotulinumtoxinA, 2/142 subjects [1.4\%]; abobotulinumtoxinA, $1 / 75$ subjects $[1.3 \%]$ ), and post-hoc analysis did not reveal significant differences (all $\mathrm{p}=1.000)$.

Within the Mexican subject population, 17/101 subjects (16.8\%) experienced an AE without causal relationship; most events were classed as 'infections and infestations' $(n=6)$ or 'cardiac disorders' $(\mathrm{n}=5)$. One subject experienced two ADRs (urinary tract infection and lower respiratory tract infection) that were both classed as SADRs; however, the relationship to the study treatment was considered 'not assessable.' ADRs/AEs were recorded as serious in 14/17 subjects, with most classed as 'cardiac disorders' $(n=5)$, 'infections and infestations' $(n=5)$, or 'respiratory, thoracic, and mediastinal disorders' $(\mathrm{n}=3)$. A small cluster of serious cases of pneumonia ( $\mathrm{n}=6$ subjects) was observed.

Nine subjects died while participating in the study (one subject in the non-Mexican population and eight in the Mexican population; Supplemental Tab. 2). These subjects had received BoNT-A doses of 40-620 U for the treatment of upper- and lower-limb spasticity. Deaths occurred between 10 days and 7-8 months after treatment. All eight Mexican subjects who died had poor health and multiple comorbidities, including high blood pressure, type 2 diabetes mellitus, COPD, heart disease, HIV infection, and cancer, which were strong confounding factors. In all cases, the cause of death was considered to be unrelated to BoNT-A treatment.

\section{Global assessment of efficacy}

At all visits, most subjects in all three treatment groups were classed as responders according to the physicians' and the subjects' global assessment of efficacy. At Visit 2 following the first treatment cycle, for those with available data 
treated with incobotulinumtoxinA, onabotulinumtoxinA, and abobotulinumtoxin $A$, physician-assessed response rates were $95.5 \%$ (359/376 subjects), 91.3\% (95/104 subjects), and $98.4 \%$ (63/64 subjects), respectively. Similarly, subject-assessed response rates were $92.9 \%$ (353/380 subjects), 86.5\% (96/111 subjects), and 93.7\% (59/63 subjects), respectively.

Although the number of subjects with global efficacy assessment data reduced substantially from Visit 2 to the final visit, both physician-assessed and subject-assessed response rates in the recorded data remained high. Among subjects treated with incobotulinumtoxin A, onabotulinumtoxin $\mathrm{A}$, and abobotulinumtoxin A, physician-assessed response rates at the final visit were $97.4 \%$ (147/151 subjects), $87.0 \%$ (20/23 subjects), and 100\% (18/18 subjects), respectively; with similar subject-assessed response rates of $97.4 \%$ (149/153 subjects), $81.8 \%$ (18/22 subjects), and 100\% (18/18 subjects), respectively.

At Visit 2 and at the final visit, most physicians and subjects rated the efficacy of BoNT-A as 'very good' or 'good', regardless of BoNT-A formulation. However, these ratings were recorded for fewer subjects at the final visit (Fig. 1).

\section{HRQoL}

Treatment with all three BoNT-A formulations was associated with an improvement in HRQoL from injection Visit 1 (baseline), as measured on the EQ-5D VAS (Fig. 2). In subjects receiving incobotulinumtoxinA, onabotulinumtoxin A, and abobotulinumtoxinA, respectively, the mean (SD) EQ-5D VAS score was 53.4 (18.66), 56.1 (20.32), and 52.6 (18.75) at injection Visit 1, and 71.1 (20.35), 65.7 (18.52), and $67.0(15.64)$ at the final visit.

A post-hoc analysis of the change in EQ-5D VAS score from injection Visit 1 revealed statistically significant improvements for pooled data across all formulations, and for incobotulinumtoxinA ( $\mathrm{p}<0.0001$ from Visit 1 at each subsequent visit). For onabotulinumtoxinA and abobotulinumtoxinA, statistical significance was less consistent. In those receiving incobotulinumtoxin $A$, onabotulinumtoxin $A$, and abobotulinumtoxinA, respectively, the mean (SD) change in EQ-5D VAS score was 6.2 (16.19), 3.2 (16.49), and 2.4 (17.33) at Visit 2, and 18.6 (20.13) 5.9 (16.57), and $18.2(19.65)$ at the final visit.

In general, compared to Visit 2, the percentage of subjects reporting their condition as 'normal (no problems)' increased, and those reporting 'severe impairment (extreme problems)' decreased with all three BoNT-A formulations across all dimensions of the EQ-5D at the final visit (Supplemental Fig. 4). Also, from Visit 1 until 4 weeks post injection, subjects treated with incobotulinumtoxinA showed improvements in mean composite SF-12 physical and mental health scores, with less data available for meaningful analysis in onabotulinumtoxinA- and abobotulinumtoxinA-treated subjects (Supplemental Tab. 3).

\section{Discussion}

This non-interventional study investigated how BoNT-A is used to treat spasticity in treatment-naïve subjects in routine clinical practice settings in nine countries worldwide during up to eight treatment cycles, for up to 2 years. Over the entire study duration, the incidence of ADRs was low, and the rate was similar across all three BoNT-A treatment groups. Of the 16/600 non-Mexican subjects reporting ADRs, two subjects had ADRs considered by the treating physician to be 'definitely related' to BoNT-A treatment, but of a non-serious nature; however, half of the subjects had ADRs that, while a causal relationship to treatment could not be ruled out, were assessed by the treating physician as 'unlikely to be related' to BoNT-A treatment, including all reported SADRs. Eight of nine deaths reported during the study occurred in Mexican subjects. In all cases, these subjects had a history of poor health, the deaths were deemed to be unrelated to BoNT-A treatment, and multiple co-morbidities and strong confounding factors were present.

Physicians were able to document treatment efficacy using various impairment or function-based scales (e.g. Ashworth Scale, Tardieu Scale, Rivermead Scale, Functional Ambulation Classification Scale). However, many of these assessments are of academic value and are not performed in routine clinical practice, or only performed occasionally. For this reason, and to alleviate the potential effects of multinational differences in approval status for BoNT-A dose and indication, efficacy was evaluated using global assessments of more relevance to real-world clinical practice.

Global assessments of efficacy and tolerability showed that BoNT-A spasticity treatment was effective and well-tolerated, confirming the positive findings from previous clinical studies of BoNT-A injections for spasticity treatment, including randomised controlled trials [11-20]. No formal statistical analyses of the data were conducted; however, there did not appear to be any major differences in efficacy between BoNT-A formulations. Most subjects and physicians rated the efficacy of the first and last treatments as 'very good' or 'good' in all three BoNT-A treatment groups, with no noticeable reduction in perceived benefit with repeated treatment over time. Also, assessment of HRQoL, based on EQ-5D VAS, suggested that BoNT-A treatment improved HRQoL for the duration of the study, i.e. up to 2 years. However, when considering the apparent gradual increase in HRQoL scores over time, the effects of selection bias, resulting from the diminishing analysis population, should be taken into account.

It is worth underlining that physicians were given complete freedom to choose the BoNT-A formulation, dose and interval between injections, diagnostic and treatment procedures, and concomitant medications for each subject. The majority of participating physicians (59.1\%) stated that they would like to inject higher doses of BoNT-A than is permitted by current product labelling, suggesting that many felt that current 


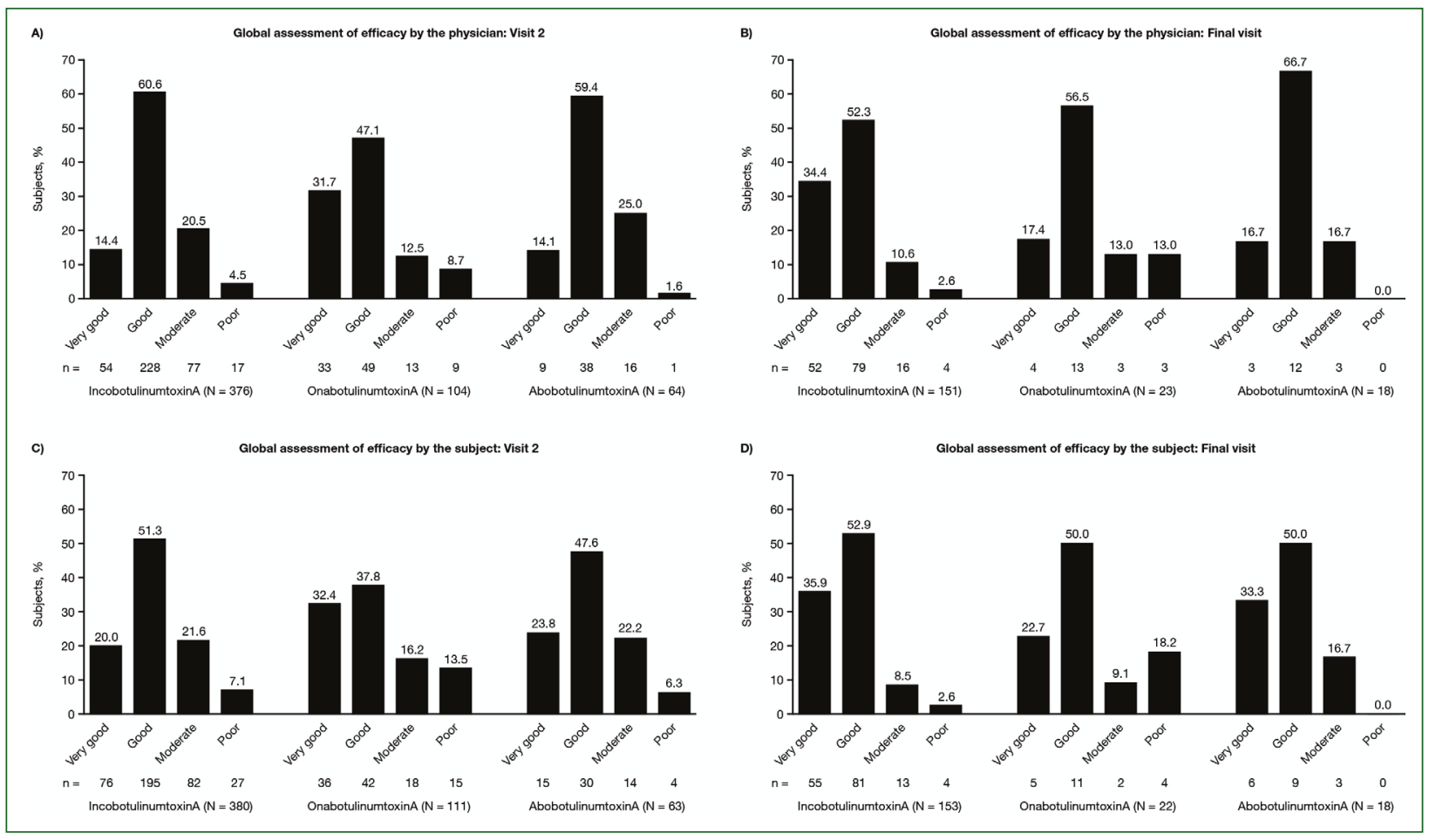

Figure 1. Proportion of subjects with very good, good, moderate, or poor global assessment of efficacy ratings according to physician at A) Visit 2 (evaluation of treatment cycle 1) and B) final visit (evaluation of treatment cycle 8), and according to subject at C) Visit 2 and D) final visit ${ }^{a}$

${ }^{a}$ Final visit occurred at end of study, subjects did not receive an injection at this point and only those who returned for assessment were included in analysis

Percentages are based on non-missing values; $\mathrm{N}$ - total number of subjects assessed

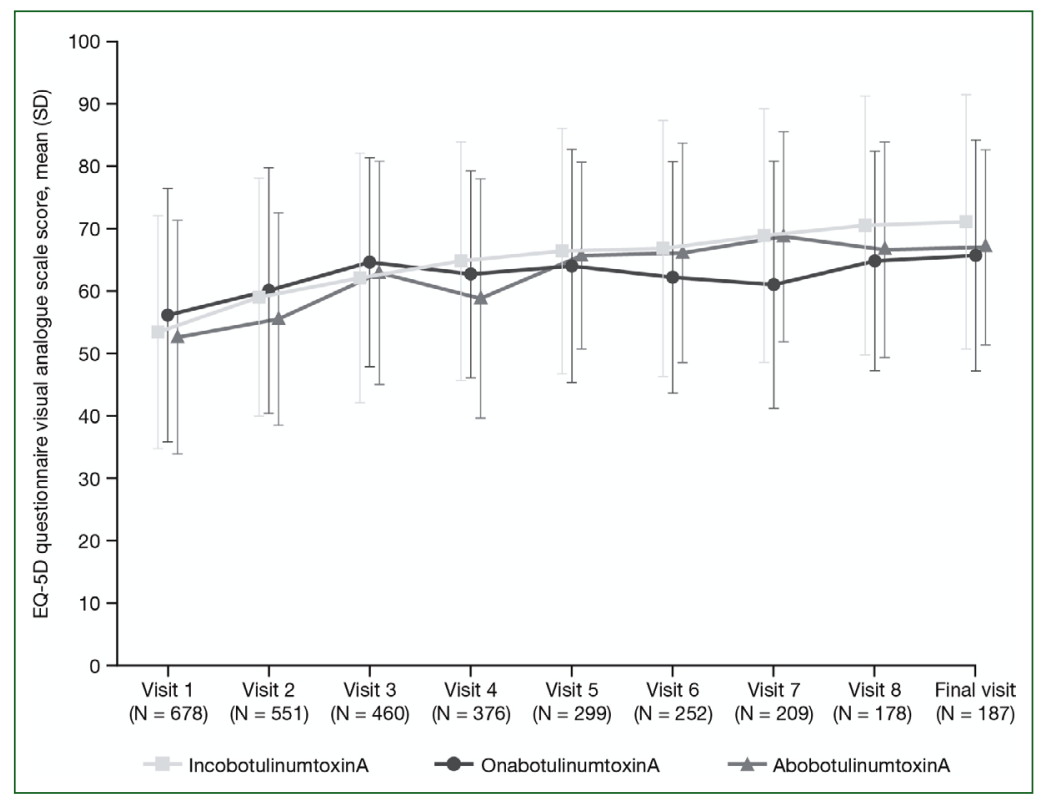

Figure 2. Mean (SD) HRQoL (EQ-5D visual analogue scale score)

Final visit occurred at end of study, subjects did not receive an injection at this point and only those who returned for assessment were included in analysis

EQ-5D - EuroQoL 5-dimensions; HRQoL - health-related quality of life 
maximum dose recommendations might be too restrictive for some subjects' needs (see relevant prescribing information for details of current indicated doses of BoNT-A products [5-10]). This is consistent with a survey reporting that a physician-estimated $24.6 \%$ of individuals could benefit from higher doses of BoNT-A, and that lifting dosing and interval restrictions could improve therapy outcomes and treatment satisfaction [27]. A prospective, dose-titration study investigated the safety and efficacy of escalating incobotulinumtoxinA doses in subjects with upper- and lower-limb spasticity [20]. Incobotulinumtoxin $\mathrm{A}$ doses up to $800 \mathrm{U}$ were well tolerated and allowed the treatment of a greater number of muscles and clinical patterns in a single treatment cycle, which may alleviate physicians' requirements to prioritise clinical patterns for treatment.

The strengths of this study include the large population $(\mathrm{n}=701$ for the safety analysis) from nine countries worldwide, the long duration (up to 2 years), and the fact that all participating subjects were treatment-naïve at study entry.

However, the representation of routine clinical practice across nine countries with different licenced indications and recommendations could also be viewed as a limitation. Further limitations include the lack of subject-reported safety information, and the gradual attrition of subject information and the resulting convenience sampling at later treatment cycles. Such attrition may seem discordant with the reported efficacy; additional studies are needed to investigate the factors contributing to treatment discontinuation in those who experience efficacy with BoNT-A. Although not reported in this study, reasons for attrition in such non-interventional studies can include subjects being lost to follow-up, either because they do not return for treatment or because the physician no longer documents the data, as well as when subjects experience treatment dissatisfaction, or indeed improvement in their symptoms, and do not require further treatment.

\section{Conclusion}

These results support the routine use of BoNT-A therapy in adults with spasticity. Throughout this 2-year real-world study, BoNT-A treatment was well tolerated, effective, and associated with an improved HRQoL.

\section{Clinical implications/future directions}

The results of the SPACE study make a valuable contribution to the broader understanding of how physicians use BoNT-A therapy to manage spasticity in real-world clinical practice. Further studies are required to investigate any correlations in practice and outcomes across the participating countries, including the effects of guidance techniques known to influence the efficacy of BoNT treatment [28].

Furthermore, research would be welcomed that brings clinicians closer to a consensus about objective outcome measures which could be used to capture the diverse range of benefits in subjects with spasticity, including the evaluation of goal attainment and functional improvements included in previous trials $[20,24,29]$.

Acknowledgements: The authors wish to thank the subjects and study investigators, and Kati Sternberg, PhD, for her input into the study and manuscript development. Medical writing support, under the direction of the authors, was provided by Claire Cairney, PhD, CMC Connect, McCann Health Medical Communications, funded by Merz Pharmaceuticals $\mathrm{GmbH}$, in accordance with Good Publication Practice (GPP3) guidelines. Conflicts of interest: $J H$ and $C C B$ have no conflict of interest to declare. NR has served as a consultant for Allergan and Merz Pharmaceuticals. SK received training fees and meeting sponsorships from Allergan, Ipsen, and Merz Pharmaceuticals, and has received compensation from Ipsen for conducting clinical trials. PS received honoraria and fees from Allergan for presentations at meetings and congresses. SH has served as a consultant for Allergan, Ipsen, and Merz Pharmaceuticals. OS was an employee of Merz Pharmaceuticals GmbH at the time the study was conducted. CKF and GC are employees of Merz Pharmaceuticals GmbH. WHJ has served as a consultant for Abbvie, Allergan, Bial, Ipsen, Merz Pharmaceuticals, UCB, and Zambon.

Funding: This study was supported by Merz Pharmaceuticals GmbH, Frankfurt am Main, Germany.

\section{References}

1. Esquenazi A, Novak I, Sheean G, et al. International consensus statement for the use of botulinum toxin treatment in adults and children with neurological impairments-introduction. Eur J Neurol. 2010; 17 Suppl 2: 1-8, doi: 10.1111/j.1468-1331.2010.03125.x, indexed in Pubmed: 20633176.

2. Royal College of Physicians, British Society of Rehabilitation Medicine, Chartered Society of Physiotherapy, Spasticity in adults: management using botulinum toxin. National guidelines 2018 https://www rcplondon.ac.uk/sites/default/files/documents/spasticity-in-adults-management-botulinum-toxin.pdf. Accessed October 2, 2019.

3. Simpson DM, Hallett M, Ashman EJ, et al. Practice guideline update summary: Botulinum neurotoxin for the treatment of blepharospasm, cervical dystonia, adult spasticity, and headache: Report of the Guideline Development Subcommittee of the American Academy of Neurology. Neurology. 2016; 86(19): 1818-1826, doi: 10.1212/ WNL.0000000000002560, indexed in Pubmed: 27164716.

4. Wissel J, Ward AB, Erztgaard P, et al. European consensus table on the use of botulinum toxin type A in adult spasticity. J Rehabil Med. 2009; 41(1): 13-25, doi: 10.2340/16501977-0303, indexed in Pubmed: 19197564.

5. Merz Pharmaceuticals LLC. Highlights of Prescribing Information Xeomin $^{\circledast}$. 2019. https://www.accessdata.fda.gov/drugsatfda_docs/ label/2019/125360s074lbl.pdf. Accessed May 17, 2019

6. Merz Pharma UK Ltd. Xeomin ${ }^{\circledR}(50 / 100 / 200)$ Summary of Product Characteristics. 2020. https://www.medicines.org.uk/emc/product/2162/smpc. Accessed March 19, 2020.

7. Allergan Inc. Highlights of Prescribing Information - Botox 2019. https://www.accessdata.fda.gov/drugsatfda_docs/label/2019/ 103000s5309lbl.pdf. Accessed June 3, 2019. 
8. Allergan Ltd. Botox 100 U Summary of Product Characteristics. 2020. https://www.medicines.org.uk/EMC/medicine/112/SPC/. Accessed March 19, 2020.

9. Ipsen Biopharm Ltd. Highlights of Prescribing Information - Dysport ${ }^{\oplus}$. 2019. https://www.accessdata.fda.gov/drugsatfda_docs/ label/2019/125274s115lbl.pdf. Accessed October 16, 2020.

10. Ipsen Biopharm Ltd. Dysport ${ }^{\oplus} 500$ U Summary of Product Characteristics. 2020. https://www.medicines.org.uk/emc/medicine/32114. Accessed March 19, 2020.

11. Bakheit AMO, Fedorova NV, Skoromets AA, et al. The beneficial antispasticity effect of botulinum toxin type $A$ is maintained after repeated treatment cycles. J Neurol Neurosurg Psychiatry. 2004; 75(11): 1558-1561, doi: 10.1136/jnnp.2003.035139, indexed in Pubmed: 15489387.

12. Gordon MF, Brashear A, Elovic E, et al. BOTOX Poststroke Spasticity Study Group. Repeated dosing of botulinum toxin type A for upper limb spasticity following stroke. Neurology. 2004; 63(10): 1971-1973, doi: 10.1212/01.wnl.0000144349.95487.91, indexed in Pubmed: 15557529.

13. Elovic EP, Brashear A, Kaelin D, et al. Repeated treatments with botulinum toxin type A produce sustained decreases in the limitations associated with focal upper-limb poststroke spasticity for caregivers and patients. Arch Phys Med Rehabil. 2008; 89(5): 799-806, doi: 10.1016/j.apmr.2008.01.007, indexed in Pubmed: 18452724.

14. Kaňovský P, Slawek J, Denes Z, et al. Efficacy and safety of botulinum neurotoxin NT 201 in poststroke upper limb spasticity. Clin Neuropharmacol. 2009; 32(5): 259-265, doi: 10.1097/ WNF.0b013e3181b13308, indexed in Pubmed: 19644361.

15. Kaji R, Osako Y, Suyama K, et al. GSK1358820 Spasticity Study Group. Botulinum toxin type A in post-stroke lower limb spasticity: a multicenter, double-blind, placebo-controlled trial. J Neurol. 2010; 257(8): 1330-1337, doi: 10.1007/s00415-010-5526-3, indexed in Pubmed: 20358216.

16. Kaňovský P, Slawek J, Denes Z, et al. Efficacy and safety of treatment with incobotulinum toxin A (botulinum neurotoxin type A free from complexing proteins; NT 201) in post-stroke upper limb spasticity. J Rehabil Med. 2011; 43(6): 486-492, doi: 10.2340/165019770796, indexed in Pubmed: 21533328.

17. Gracies JM, Brashear A, Jech R, et al. Safety and efficacy of abobotulinumtoxinA for hemiparesis in adults with upper limb spasticity after stroke or traumatic brain injury: a double-blind randomised controlled trial. Lancet Neurol. 2015; 14(10): 992-1001, doi: 10.1016/s14744422(15)00216-1.

18. Elovic EP, Munin MC, Kaňovský P, et al. Randomized, placebo-controlled trial of incobotulinumtoxin A for upper-limb post-stroke spasticity. Muscle Nerve. 2016; 53(3): 415-421, doi: 10.1002/mus.24776, indexed in Pubmed: 26201835.

19. Wein T, Esquenazi A, Jost WH, et al. OnabotulinumtoxinA for the treatment of poststroke distal lower limb spasticity: a randomized trial. PM R. 2018; 10(7): 693-703, doi: 10.1016/j.pmrj.2017.12.006, indexed in Pubmed: 29330071.
20. Wissel J, Bensmail D, Ferreira JJ, et al. TOWER study investigators. Safety and efficacy of incobotulinumtoxinA doses up to $800 \mathrm{U}$ in limb spasticity: The TOWER study. Neurology. 2017; 88(14): 13211328, doi: 10.1212/WNL.0000000000003789, indexed in Pubmed: 28283596.

21. Bakheit AM, Zakine B, Maisonobe P, et al. The profile of patients and current practice of treatment of upper limb muscle spasticity with botulinum toxin type A: an international survey. Int J Rehabil Res. 2010; 33(3): 199-204, doi: 10.1097/MRR.0b013e328332f5e0, indexed in Pubmed: 20154631.

22. Mohammadi B, Balouch SA, Dengler R, et al. Long-term treatment of spasticity with botulinum toxin type A: an analysis of 1221 treatments in 137 patients. Neurol Res. 2010; 32(3): 309-313, doi: 10.1179/016164109X12478302362734, indexed in Pubmed: 19726016.

23. Sarzyńska-Długosz I, Szczepańska-Szerej A, Drużdż A, et al. Real-world effectiveness of abobotulinumtoxinA (Dysport ${ }^{\circledR}$ ) in adults with upper limb spasticity in routine clinical practice: an observational study. Neurol Neurochir Pol. 2020; 54(1): 90-99, doi: 10.5603/PJNNS. a2020.0004, indexed in Pubmed: 31956971.

24. Turner-Stokes L, Fheodoroff $K$, Jacinto J, et al. Results from the Upper Limb International Spasticity Study-II (ULIS-II): a large, international, prospective cohort study investigating practice and goal attainment following treatment with botulinum toxin A in real-life clinical management. BMJ Open. 2013; 3(6): e002771, doi: 10.1136/bmjopen-2013-002771, indexed in Pubmed: 23794582.

25. EuroQol Group. EuroQol-a new facility for the measurement of health-related quality of life. Health Policy. 1990; 16(3): 199-208, doi: 10.1016/0168-8510(90)90421-9, indexed in Pubmed: 10109801.

26. Dressler D, Bigalke H. Pharmacology of botulinum toxin drugs. In: Truong D, Dressler D, Hallett M, editors. Manual of Botulinum Toxin Therapy. Cambridge, New York: Cambridge University Press; 2009. p.13-22, doi: 10.1017/cbo9780511575761.005.

27. Bensmail D, Hanschmann A, Wissel J. Satisfaction with botulinum toxin treatment in post-stroke spasticity: results from two cross-sectional surveys (patients and physicians). J Med Econ. 2014; 17(9): 618625, doi: 10.3111/13696998.2014.925462, indexed in Pubmed: 24841450.

28. Santamato A, Micello MF, Panza F, et al. Can botulinum toxin type A injection technique influence the clinical outcome of patients with post-stroke upper limb spasticity? A randomized controlled trial comparing manual needle placement and ultrasound-guided injection techniques. J Neurol Sci. 2014; 347(1-2): 39-43, doi: 10.1016/j. jns.2014.09.016, indexed in Pubmed: 25263601.

29. Ward AB, Wissel J, Borg J, et al. Functional goal achievement in post-stroke spasticity patients: The Botox ${ }^{\oplus}$ Economic Spasticity Trial (BEST). J Rehabil Med. 2014; 46(6): 504-513, doi: 10.2340/16501977-1817.

30. Ware J, Jr., Kosinski M, Keller SD. A 12-Item Short-Form Health Survey: construction of scales and preliminary tests of reliability and validity. Med Care. 1996; 34(3): 220-233, doi: 10.1097/00005650199603000-00003, indexed in Pubmed: 8628042. 